\title{
A New Approach to Brainstorming: Multitouch Brainstorming
}

\author{
Sophia Atzeni \\ s.e.atzeni@tue.nl
}

\author{
Annemiek van Drunen \\ a.v.drunen@tue.nl
}

\author{
Aljosja Jacobs \\ a.p.a.jacobs@tue.nl
}

\author{
Dirk Verhagen \\ d.verhagen@tue.nl
}

Eindhoven University of Technology, the Netherlands

\begin{abstract}
Whilst researching directions to improve co-creation between knowledge workers using technology, it was found that brainstorming was an activity that might be improved upon. We designed an interactive system using multitouch technology. We have tried to leverage the advantages of such a system, especially during idea clustering. We have developed a video prototype of our system and validated the concept using focus groups. Feedback indicated an improvement over their current way of working.
\end{abstract}

Brainstorm, Multi-touch Wall, Collaboration, Knowledge Workers

\section{INTRODUCTION}

A knowledge worker's main activity and strength is creating ideas; which is often supported by cocreation behavior (Kidd, 1994). The process used to get in the proper frame-of-mind and to access creativity is brainstorming. We have designed a system which moves this, traditionally analogue, activity into the digital realm. Brainstorming is a cocreation technique used in many different fields, in many different professions, and by many different people all over the world. Yet brainstorming always has the same goal: generating ideas to find a solution to a challenge. To attain this goal the most important rules are to think divergent when generating ideas. However, to end up with a feasible solution you need to start thinking convergent when evaluating the ideas (Osborn, 1957). Traditionally, post-its are used to generate ideas, they can be moved to cluster these ideas, and an evaluation is needed to compare ideas, combine ideas, and filter out the best ideas.

Digital systems could enhance the brainstorming process. Brainstorming often produces many ideas, reasoning, and mind-map like structures. In addition, it is also often a lengthy process. Because of this, saving and loading of relevant data could offer significant advantages over using only paper. There are also factors in a brainstorm that can be important during the process, which are not always explicitly available to the participants (e.g. the number of ideas generated per minute, which idea belongs to whom, the time a brainstorm may take).
A digital solution could analyze these factors and make intelligent decisions based on these. For example, when the idea rate drops down, a automatic generated creative cue can help the participants starting to generate ideas again.

Seeing as how brainstorms usually involve a public display of ideas, it seemed a natural fit to use a large interactive display. As mode of interaction we choose a multitouch screen because of its egalitarian input qualities (Wigdor et al., 2009). Multitouch technology is far from a novel technology. While it offers large possibilities for new interface paradigms, as well as promise in the field of multi-user devices, we are still very much unaware of what specific applications may aid us in our everyday life. So far, the application many people are familiar with is the 'photos application', but we feel that especially in the future workspace many possibilities are left unexplored. Therefore, we explored how a multitouch wall can be used for collaborative activities in the workspace in general and brainstorming specific.

\section{PROCESS}

To involve the knowledge workers in the design development as much as possible, a user-centered approach was chosen. To identify the main collaboration problems between knowledge workers we started with contextual interviews and observations. From these two methods several issues were derived that knowledge workers experience during collaboration. Together with 
several brainstorm sessions within the team, those issues were used as inspiration for some initial concepts. The concepts were translated into two movie prototypes with contrasting design choices. In a focus group with knowledge workers the movie prototypes were shown to elicit discussion and an evaluation on the contrasting design choices. In addition, the same method was used for expert reviews. The feedback received was used to reiterate our first concepts into a more definite concept. A definite movie prototype was developed for another focus group evaluation with knowledge workers and expert reviews. During the final focus group, users' opinions were also gathered using a small questionnaire; the USE questionnaire (Lund, 2001). Within this standardized usability questionnaire participants rated a 5-point Likert scale about the brainstorm application compared to their normal brainstorms. Items were rated on the topics of usefulness, ease of use, and satisfaction. In both focus groups we had a mix of various professions, ranging from healthcare to management to ICT. For the expert reviews, we used industrial designers experienced and trained in carrying out brainstorms. Finally, the feedback received from these sources was used for requirements and recommendations when designing a brainstorm application for a multitouch wall (MTW).

\section{DESIGN ISSUES}

Before committing to the final concept we had a list of issues surrounded by some controversy. As described we created two movie prototypes with contrasting choices. The underlying design issues with these choices were: ownership of ideas, method of idea input, visualization of generated ideas, the idea generation rate, individual contribution to the brainstorm, using creative cues, exploring ideas with games, method of clustering, creation of clusters, evaluation method, accountability for votes, sociometric feedback and last, a voting game. Sociometric feedback means that it is made visible if one person is domination the discussion e.g. by displaying on the MTW how much someone is talking (Kim et al. 2008). We created two prototypes with contrasting features. Each prototype was shown to a focus group in its entirety, after which individual features where discussed (and the relevant portion of movie was shown again).
Table 1: comparison of different issues between the two prototypes (Prototype A and Prototype B).

\begin{tabular}{|c|c|}
\hline Prototype A & Prototype B \\
\hline Ideas are anonimized & $\begin{array}{l}\text { It is visible who came up with } \\
\text { which idea }\end{array}$ \\
\hline $\begin{array}{l}\text { Input takes place at a } \\
\text { table }\end{array}$ & Input is done at the MTW \\
\hline $\begin{array}{l}\text { A 'flow' of ideas was } \\
\text { visible }\end{array}$ & $\begin{array}{l}\text { Ideas are visible as a } \\
\text { collection of post-its, much } \\
\text { like classic brainstorming }\end{array}$ \\
\hline $\begin{array}{lc}\text { An } & \text { aggregated } \\
\text { \#ideas/min } & \text { score is } \\
\text { shown } & \end{array}$ & \#Ideas/min is not shown \\
\hline $\begin{array}{l}\text { No individual score is } \\
\text { shown }\end{array}$ & $\begin{array}{l}\text { Your individual contribution } \\
\text { in \#ideas is shown }\end{array}$ \\
\hline $\begin{array}{l}\text { Creative cues are given } \\
\text { when \#ideas/min goes } \\
\text { down }\end{array}$ & $\begin{array}{l}\text { No creative cues are ever } \\
\text { shown }\end{array}$ \\
\hline There are no games & $\begin{array}{l}\text { There are games to } \\
\text { explore/explain ideas }\end{array}$ \\
\hline $\begin{array}{l}\text { Classic clustering: } \\
\text { putting post-its together } \\
\text { in groups }\end{array}$ & 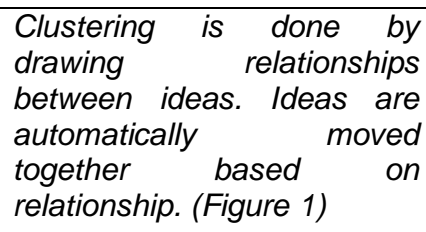 \\
\hline $\begin{array}{l}\text { Board detects when } \\
\text { there is a cluster and } \\
\text { makes them more salient } \\
\text { as they remain } \\
\text { unchanged. }\end{array}$ & $\begin{array}{l}\text { Users draw } \\
\text { themselves }\end{array}$ \\
\hline $\begin{array}{l}\text { There was no compare } \\
\text { tool to help users } \\
\text { evaluate }\end{array}$ & $\begin{array}{l}\text { Ideas are discussed in depth } \\
\text { with a compare tool (Figure } \\
\text { 2) }\end{array}$ \\
\hline $\begin{array}{l}\text { It is visible who votes on } \\
\text { what }\end{array}$ & $\begin{array}{l}\text { Votes are shown when } \\
\text { everyone is done }\end{array}$ \\
\hline $\begin{array}{l}\text { There is no Sociometric } \\
\text { feedback }\end{array}$ & $\begin{array}{l}\text { Sociometric feedback is } \\
\text { given }\end{array}$ \\
\hline There is no voting game & $\begin{array}{l}\text { There is a game used to vote } \\
\text { (pig-feeding) }\end{array}$ \\
\hline
\end{tabular}

\subsection{Conclusions}

Participants were very clear about issues in the idea generation phase. They preferred inputting ideas from the table. In their eyes this created a much more relaxed atmosphere. Ideas should be visible on the wall in a non-anonymous fashion. In this way they can trigger new ideas. A suggestion was to fade older ideas. There should be no explicit mechanism to indicate number of ideas per minute. There are sufficient visual and audio cues for participants to give them a sense of this. Creative cues were experiences as very positively, though 
perhaps one might want to summon them instead of generating them automatically.

A game in the exploration phase was also considered very positive. It fit the atmosphere perfectly. The downside could be participants need their own pace and method, hence it should be optional.

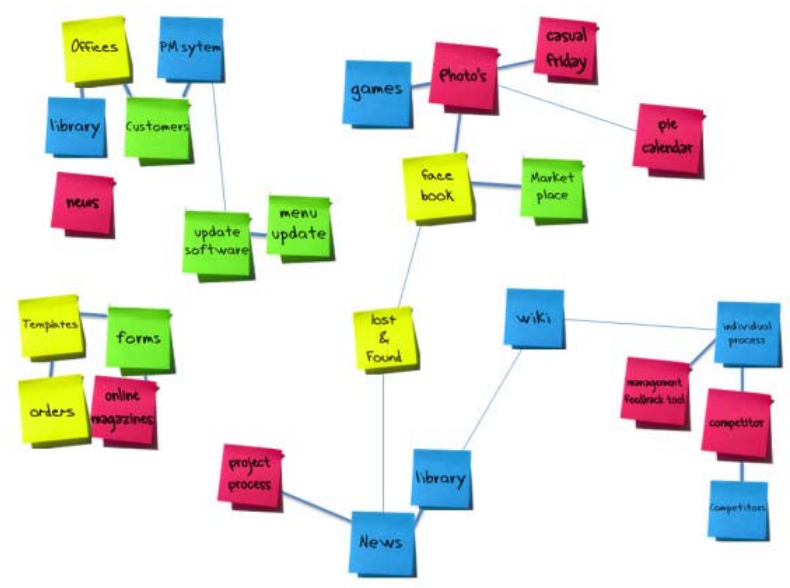

Figure 1: An example of relationship clustering

There was no clear preference for method of clustering. Participants agreed classic clustering and relationship clustering do not exclude each other, and the ideas could perhaps be combined to form a more powerful clustering method, yet still close to their mental model of clustering. Classic clustering could take place first with relationship clustering filling in more details. A higher fidelity prototype would be needed to arrive at a deeper understanding of these issues. As to the matter of implicit clustering, users liked it, but were afraid a system would get this wrong too often, hence the option to explicitly create clusters should always be present.

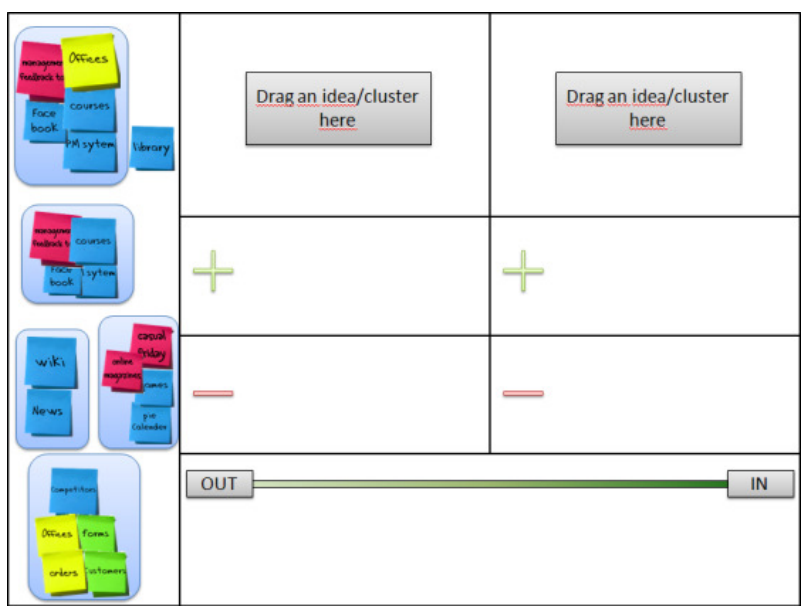

Figure 2: The compare view
In the idea evaluation view it turned out that the compare view was considered a useful tool, but not in this linear matter. Users should be able to invoke this tool whenever needed, with the ideas still visible. Lightening the voting process with a game was seen as a very positive thing, as usually this is a tense phase during the brainstorm. In addition, participants thought it was important to track votes individually, so you can explain your reasoning. Sociometric feedback was seen as a bit too confronting and patronizing. While original research by Kim et al. show improvements in group conversations, despite what user's opinions may be, we decided to not include it in the final concept as we did not want to focus participants on this.

\section{CONCEPT DESCRIPTION}

The input for this concept was inspired by the observations and interviews conducted with knowledge workers. We found out that groups often lack structure in their brainstorm; clustering and evaluations were skipped ending up only with a page full of unstructured ideas. Users felt their individual opinions were not always taken into account. Furthermore, people lacked insight into how they arrived at various ideas or ideas got lost during the process. In addition, arguments were not well recorded or weighed in when it was time to make a decision. Finally, brainstorms structured as formal meetings resulted in stifling creativity. These mishaps in brainstorming were found across a number of different organizations.

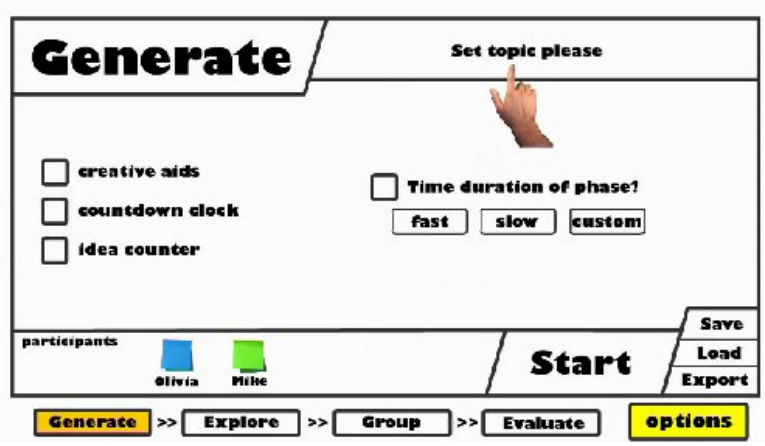

Figure 3: A set-up screen for the idea generation phase

An important design decision was to entice the brainstorm group to conduct a complete structured brainstorm by guiding them through the traditional brainstorm phases; idea generation, idea explanation (through games), clustering of ideas and evaluation of ideas. In these phases, both the processes of diverging and then converging ideas are facilitated, which are fundamental parts of a brainstorm. Although the brainstorm application offers more structure over its analogue counterpart, the flexibility and freedom to change and shift from the different phases is enhanced by being able to 
save and load between the phases. We placed setup screens before each phase to allow users to customize their brainstorm experience (Figure 3). Another important aspect is the fact that the first game draws users from the table to the MTW after the idea generation phase. In this way users are playfully moved towards the area and can gradually get used to the interaction with a wall.

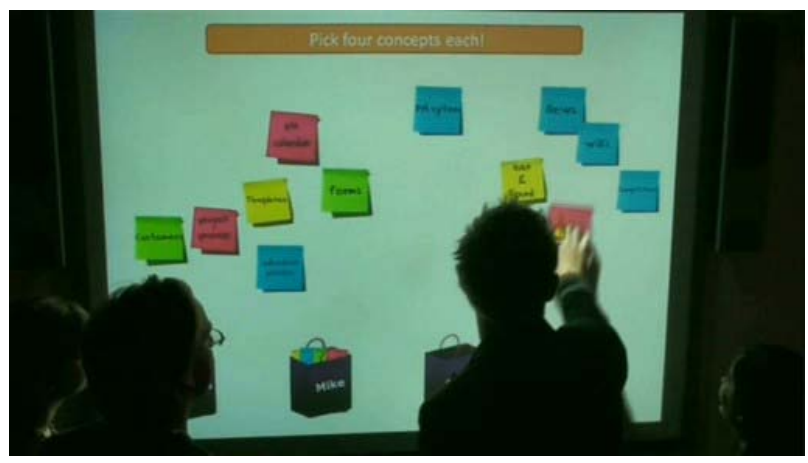

Figure 4: Movie prototype of brainstorm application

We try to get users to play with their ideas, without feeling limited by matters such as screen size, fear of not being able to go back to previous versions, or the amount of effort involved in restructuring ideas. In addition, we leverage the advantages of a large interactive display, such as a MTW, in enabling users to create more structure in their 'cloud of ideas' and represent their ideas in a dynamic way. Another advantage of using a MTW is that the wall affords a shared view for all brainstorm users. Within the several brainstorm phases, easy to use operations are possible; resize, undo, moving, drawing lines and copy/pasting and moving digital post-its as well as entire clusters are supported. All these operations can be carried out with natural gestures (figure 4).

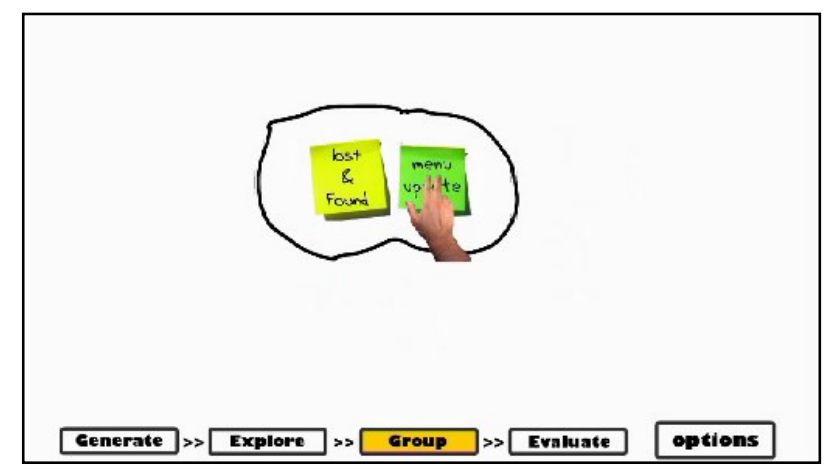

Figure 5: A bubble-cluster

The clustering phase specifically was redesigned, combining the ideas of relationship clustering with classic clustering. They can create clusters by drawing a circle around a group of post-its (figure 5). These circles function like bubbles. When a user drags an object (post-it or cluster) in or out of a bubble, the cluster-border gives some resistance before the object moves through the cluster-border. This bubble metaphor was designed to make clustering more playful. Furthermore relations could now be drawn between ideas, next to normal classic clustering. We preserved the mechanism where ideas would move closer together based on the strength of their relationship (which could be customised).

The games were preserved in the final concept. Between generating ideas and clustering ideas we would have a game where users would have to quickly grab four ideas each to explain, or pick the odd idea out of a set of four. For voting we used a game where users would have to feed pigs, where pigs represented ideas.

\section{DISCUSSION AND RECOMMENDATIONS}

After aggregating the results from the final focus group and expert review we arrived at three main recommendations. These recommendations are important when constructing a multitouch brainstorm application such as this.

First, the flow in the process should be dynamic. The system should never 'force' a user down a path. Users found this one of the main strengths of a digital application, as it could move from the end of a brainstorm back to the beginning without any hassle at all. In this way decisions were retraceable. Second, the fun atmosphere was considered a very important factor, but also one which could detract from the serious nature of a brainstorm, according to participants. Games with a clear brainstorm-objective were seen as balanced ways to make the process more light-hearted, as the objective was serious. The pig-game was seen as most fun, followed by the bubble-like clustering. Third, we found that ideas should be visible and interactive during all the phases of the brainstorm, especially experts were very adamant on this issue. One of the benefits a digital system offers is dynamically restructuring the representation of ideas, while maintaining and possibly switching to alternative representations. These representations can be used for different activities in the brainstorm, such as voting, using a decision support system or other supporting structures.

Some open issues still remain. While the clustering design was seen as more fun and very useful, it remains to be seen if users would use all the added features in a real-life brainstorm. We believe that with carefully designed low-level interactions, users could do this. In addition, we were not quite sure if sociometric feedback would enhance the process. Research shows it improves group conversations 
and decision making in a meeting-like setting, yet our participants were doubtful on the added value. It remains to be seen how well this idea can be adopted in evaluation of brainstorm ideas. Furthermore it would be interesting to see if more divergent, or simply just more ideas, over a traditional brainstorm, would be generated using such a tool. A large test where multiple groups of people would use a digital tool, versus groups of people without the digital enhancement would be needed.

Overall, users indicated that a digital way of brainstorming would be more fun, easy to use and more stimulating in a creative sense, than their current way of idea-generation. Not only did they comment on this in the final focus group, but the results from the USE questionnaire showed the same trend (based on a small sample however), with all items scoring 4 or higher. While these results are based mostly on subjective methods, it does indicate knowledge workers from various fields-of-work are enthusiastic about such an initiative, and do not seem hesitant in enhancing traditional analogue brainstorming.

Using relatively simple interactions and ideas, on hardware which is quickly becoming more mainstream, one can create a way-of-working for which there is much enthusiasm and which shows much promise. A digital brainstorm shows an added value over regular brainstorming and could become a valuable addition to the future workspace.

\section{REFERENCES}

Kidd, A. (1994) The Marks are on the Knowledge Worker. In Proceedings of CHI'94 Human Factors in Computing Systems, Boston, 1994, 186-191. ACM Press, New York.

Kim, T., A. Chang, L. Holland, and A. Pentland. (2008). Meeting mediator: enhancing group collaborationusing sociometric feedback. Proceedings of the ACM Conference on Computer Supported Cooperative Work. New York, NY, 2008. 457-466.

Lund, A. (2001). USE questionnaire. Usability and User Experience. 8(2).

Osborn, A.F. (1957). Applied Imagination: Principles and Procedures. Charles Scribner's Son, New York

Wigdor, D., Jiang, H., Forlines, C., Borkin, M., Shen,C. (2009). The WeSpace: The Design, Development and Deployment of a Walk-Up and
Share Multi-Surface Visual Collaboration System. Proceedings of $\mathrm{CHI}$ 2009, Boston, MA

\section{ACKNOWLEDGMENTS}

Our research project was carried out in collaboration with the future workspaces project from Novay (www.futureworkspaces.nl/about) We would like to thank Ruud Janssen for his excellent guidance. Also a big thank you to Geke Ludden, for the smooth collaboration. Furthermore, a word of thanks to all the participants for providing us with valuable feedback

Compilation of final brainstorm concept: http://www.youtube.com/watch?v=Ff2wFCZLj_I 